\title{
"I run the world's largest historical outreach project and it's on a cesspool of a website." Moderating a public scholarship site on Reddit: A case study of $r$ /AskHistorians
}

\author{
SARAH A. GILBERT, University of Maryland, United States
}

Online communities provide important functions in their participants' lives, from providing spaces to discuss topics of interest to supporting the development of close, personal relationships. Volunteer moderators play key roles in maintaining these spaces, such as creating and enforcing rules and modeling normative behavior While these users play important governance roles in online spaces, less is known about how the work they do is impacted by platform design and culture. r/AskHistorians, a Reddit-based question and answer forum dedicated to providing users with academic-level answers to questions about history provides an interesting case study on the impact of design and culture because of its unique rules and their strict enforcement by moderators. In this article I use interviews with $\mathrm{r}$ /AskHistorians moderators and community members, observation, and the full comment log of a highly upvoted thread to describe the impact of Reddit's design and culture on moderation work. Results show that visible moderation work that is often interpreted as censorship, and the default masculine whiteness of Reddit, create challenges for moderators who use the subreddit as a public history site. Nonetheless, r/AskHistorians moderators have carved a space on Reddit where, through their public scholarship work, the community serves as a model for combating misinformation by building trust in academic processes.

CCS Concepts: • Human-centered computing $\rightarrow$ Computer supported cooperative work.

Additional Key Words and Phrases: moderation, invisible work, Reddit, motivation, online communities, public scholarship

ACM Reference Format:

Sarah A. Gilbert. 2020. "I run the world's largest historical outreach project and it's on a cesspool of a website." Moderating a public scholarship site on Reddit: A case study of r/AskHistorians. 1, 1 (February 2020), 27 pages. https://doi.org/10.1145/nnnnnnn.nnnnnnn

\section{INTRODUCTION}

On April 26th 2017, a Reddit user posed a question to the subreddit, r/AskHistorians, an online community dedicated to providing in-depth answers to historical questions. This question became the fifth most popular question asked on the subreddit of all time, receiving 15,023 upvotes. It asked, "What was the context to these photos and was it uncommon (NSFW)[not safe for work]?" The body of the post contained the following text:

Came across these photos on a porn gallery website. Without clicking, the photos depict a sex show of at least three women performing various acts in front of men in uniform.

Author's address: Sarah A. Gilbert, sagilber@umd.edu, University of Maryland, College Park, Maryland, United States.

Permission to make digital or hard copies of all or part of this work for personal or classroom use is granted without fee provided that copies are not made or distributed for profit or commercial advantage and that copies bear this notice and the full citation on the first page. Copyrights for components of this work owned by others than ACM must be honored. Abstracting with credit is permitted. To copy otherwise, or republish, to post on servers or to redistribute to lists, requires prior specific permission and/or a fee. Request permissions from permissions@acm.org.

(c) 2020 Association for Computing Machinery.

XXXX-XXXX/2020/2-ART \$15.00

https://doi.org/10.1145/nnnnnnn.nnnnnnn

, Vol. 1, No. 1, Article . Publication date: February 2020. 
I'm not sure what kind of troops they are, what era this is, or even if all the photos are from the same show (does not appear that way).

Is there any context to this? And in that context, was this sort of thing common at all? Were they sanctioned, ignored, or banned from the group watching?

The post contained a link to thirteen photographs.

The subject of the post and inclusion of sexual photographs was unlike questions typically posed to $\mathrm{r}$ /AskHistorians. The question's uniqueness, subject, and content meant that many readers were particularly interested in having the question answered. However, the question was challenging for moderators: do they remove the thread or allow it to remain? Ultimately, the decision was made to allow it to remain. But this decision came at a cost to those same moderators who were tasked with responding to the question and maintaining civility within the thread. In return, they were subjected to aggressive argumentation and abusive messages from users.

From an outsider's point of view, this question and the discussions that followed were a success. The user who posed the question received a response, and those who were engaged in the thread's discussion stated that they had learned from it. Thus, this question illustrates not only the challenges faced by moderators, but also their achievements. This study uses the full comment log of this thread, including removed comments, observation, and interviews with moderators and r/AskHistorians' users to describe the work r/AskHistorians moderators do. Findings highlight the impact of Reddit's technological design and culture on their experiences doing this work. Reddit's largely white and masculine culture, combined with technical features that visualize moderation in ways largely interpreted as censorship make the work of public history emotionally laborious and may discourage participation by those with knowledge or interests outside of Western men's history, thus creating a vicious cycle. In addition to describing the challenges faced by volunteer moderators, this paper highlights the cascading effects of their work on Reddit users and the Reddit community, and provides insight into how moderation can be leveraged to promote civil discourse, provide learning experiences, and foster trust.

This paper contains hate speech, violent speech, and references sexual violence.

\section{BACKGROUND}

\subsection{Volunteer Moderation}

An early promise of online communities was that they would provide an egalitarian space where judgements would be based on merit rather than status [40] and users could freely interact, unencumbered by the norms and expectations of offline life. While many platforms and online communities were founded on ideals of freedom, the anti-social activities that emerged, such as flaming, trolling, abuse, and harassment, necessitated the development of rules and sanctions $[19,26,30,63]$. Enforcing rules and norms requires human labor. While large platforms, such as Facebook, rely heavily on paid moderation, in smaller online communities this work is taken up by community leaders and volunteer moderators who perform the mechanisms required to facilitate prosocial behaviour and prevent antisocial behaviour [27]. On Reddit, a platform that aggregates communities called subreddits, the bulk of moderation work is conducted by volunteer moderators

There are similarities between paid and volunteer moderation - both jobs involve evaluating content, determining if it violates defined rules and standards, and making decisions about whether to keep or remove potentially offending material. However, volunteer moderators differ in that they have a level of control and self-determination that paid moderators do not [26] and they are often people who are known and interact with community members. This level of self-efficacy can be highly motivating for moderators $[60,61]$, but also results in additional work as moderators 
negotiate norms and ideals, such as democracy and meritocracy, with their role as the arbiter of their community. In spaces that value democracy and meritocracy, moderators may struggle with their role as oligarchs, where ultimately, they, rather than community members, wield control $[11,50,65,66]$. In addition to these struggles about their identity, volunteer moderators also engage with the demands of community members, platforms, and each other [53], which Matias [49-51] describes as "civic labor." Work by Matias [49-51], Lo [46], and Dosono and Semaan [20] focus on the labor of moderation. Lo [46], in her study of Twitch moderators, outlines the type of work done by volunteers, and finds that while much of the work is proactive, such as socializing and communicating with community members, the most visible work is reactive, in the form of responding to offending content. In their study of the work of moderating subreddits devoted to topics of interest to Asian and Pacific Islanders, Dosono and Semaan [20] describe the emotional labor of moderation work and the strategies moderators employ to manage it. These studies highlight the challenges moderators face as they navigate disparate expectations from various stakeholders.

In addition to personal and social conflict, moderation work is also affected by the technological constraints of the platforms on which moderators operate. Influenced by Latour's Actor Network Theory, Massanari [48] describes how Reddit's design and governance promote viewpoints held by the majority of users, thus drowning out the voices of marginalized populations as users choose what content is promoted or obfuscated through voting. Squirrell [67] elaborates on Massanari's work, highlighting how Reddit moderators use platform features and affordances, such as FAQ pages and flairs to establish trust and build consensus. Postigo [60] describes how moderation work manifests in an online environment: "In the case of the Internet, this labor is always in plain site [sic] (we see the wealth of information on the Web) yet those who do the work of generating and maintaining the Web remain hidden away under the rhetoric of volunteerism or hobby" p. 2019. What is defined as "work" often aligns with activities that involve effort. The value and legitimacy of such work is commonly tied to how visible that effort is to others. For example, when effort is unseen (such as the effort it takes to maintain a smile when serving rude customers) or is viewed as an act of love or natural behaviour (such as raising children) it may not be viewed as work and thus rendered invisible. In digital spaces, platform design can also make work visible or invisible [68]. For example, responding to a person through an instant messenger application, such as Facebook's messenger or Apple's iMessage, is visible because the person on the receiving end can see typing awareness indicators. However, the same action taken on a different platform, such as email, is not visible. Making work visible or invisible can affect how work is perceived [68]. While making work visible can provide legitimacy, as with child rearing, it may also have negative consequences, such as added surveillance that can result in additional work and dehumanization [68]. This study describes how platform technology makes moderation work visible and invisible, and how visibility affects the labor of moderation work.

\subsection{Reddit's Structure and Culture}

Because r/AskHistorians is hosted on Reddit, the following section describes what Reddit is, how it works, and explains the origins of its free speech culture. Reddit is a popular content sharing site that describes itself as 'the front page of the internet.' It is comprised of a series of interest-based communities, known as subreddits. Anyone can access content on Reddit, but content is submitted by registered users who participate pseudonymously. Creating an account can be done quickly by choosing a username and password; no authentication via email is required. Non-registered users access Reddit through its front page, also known as r/popular or r/all, which is an amalgamation of popular content from across subreddits. Registered users' default access to Reddit is through their 
home page, which is an amalgamation of popular content from across subreddits to which they have subscribed.

While content is submitted by users, its visibility is controlled by two layers of moderation: centralized and distributed. Distributed moderation describes a system where collective action by users moderates content $[42,43]$; on Reddit distributed moderation is conducted by users via voting. A primary feature of Reddit is its karma system, which describes the accumulation of points by users based on their contributions to the website. Karma points are awarded or withdrawn from submissions or comments by other users through the process of upvoting and downvoting. The total number of votes, or karma, is used to determine what content is seen; although the exact algorithm that determines which posts will be promoted to users' front page or $\mathrm{r} /$ all is proprietary, content that is highly upvoted rises to the top, while highly downvoted content is obfuscated Voting on Reddit demonstrates particular trends. In their study on information visibility during crisis events, Leavitt and Robinson [45] found that voting played a key role in dictating what information would be visible; further, information that was promoted through upvoting was also perceived to be more trustworthy while content hidden via downvotes was assumed to be less important. However, in his study comparing two subreddits devoted to the promotion of political candidates, Mills [54] found that voting patterns demonstrated bias, effectively creating a feedback loop: posts and comments would be upvoted when they confirmed the biases held by the majority of people within each subreddit, thereby making these posts and comments more visible. In turn, this visibility meant that more people were exposed to and thus upvoted the posts and comments, effectively creating echo chambers. Thus, while users may perceive upvoted content as trustworthy, that content may also reflect majority opinions and interests.

The second form of content moderation is centralized, and conducted by either paid site-level administrators or volunteer subreddit-level moderators. The majority of centralized moderation is conducted by volunteer moderators. Reddit's site-wide administrators are responsible for creating Reddit's Content Policy and penning an informal set of guidelines known as Reddiquette, while Reddit's volunteer moderators create and enforce rules that are limited to specific subreddits. Reddit rules, as well as overarching social norms, are described next.

2.2.1 Reddit's rules and norms. Rules provide explicit guidelines for behaviour while social norms reflect implicit shared understandings about actions. Social norms are often maintained cooperatively by members of a particular society through acts such as exhibiting prosocial behaviour and sanctioning antisocial behaviour [59], which requires community members to learn what behaviour is acceptable in a social space and what is not. Social norms are often learned through observation. For example, Marwick [47] describes this as one function of social surveillance, in which people observe others online and Lave and Wenger [44] describe the processes as legitimate peripheral participation, where people learn how to be part of a community. While learning norms is often passively conducted by individuals as they are enculturated into a new space, enforcing rules through sanctioning can also encourage the development of social norms [59]. Indeed, in their recent study on Reddit moderation, Jhaver, Bruckman, and Gilbert [33] found that sanctioning, in combination with explanation for the sanction, provides another method through which users can learn social norms. On Reddit, rules are created and enforced by moderators (and to a lesser extent, administrators) while norms are reflected in the behavioural patterns of users. Violating rules results in sanctioning via comment removal, or temporary or permanent bans from the subreddit. Violating norms results in sanctioning from other users via downvoting.

As Reddit is comprised of millions of subreddits, rules and norms vary throughout the site. In their study of social norms on Reddit, Chandrasekharan and colleagues [12] examined removed comments to identify multiple layers of norms on Reddit. They found that while personal attacks, misogyny, 
and hate speech were macro-level norms across Reddit, more subtle forms of discrimination, such as 'mansplaining,' mocking religion or nationality, or hostility towards immigration were meso-level norms, shared by clusters of communities. Chandrasekharan and colleagues' work is unique in that they use sanctioned content to identify social norms. Observable content, i.e., content that remains and is promoted through upvoting, reveals two Reddit-wide norms that are relevant to the current study: technoculture and free speech.

Closely tied to distributed moderation via upvoting is the value Reddit users place on freedom of speech and user-controlled content. While this is tied to design, the expectation that Reddit should serve as a bastion of free speech also arises from early site-wide norms promoted by the site's founders and administrators. From its founding in 2005 until May 2011 there were no official limitations on the kinds of content that could be shared on Reddit. The first limitation was on sharing personally identifiable information (PII), after an incident in which an alleged scammer's PII was publicized [14]. The second limitation was placed five months later, when Reddit administrators removed a subreddit in response to media exposés that $\mathrm{r}$ /jailbait, a community dedicated to posting sexually provocative pictures of young, often underage, women had been used to exchange child pornography [56]. Despite banning certain kinds of content and entire subreddits, Reddit's administration continued to follow a free speech model on the site. One year later, in a leaked communiqué to moderators, Reddit's then CEO, Yishan Wong defended free speech on Reddit, saying: "We stand for free speech. This means we are not going to ban distasteful subreddits. We will not ban legal content even if we find it odious or if we personally condemn it" [15].

Although early site-wide norms focused on the promotion of free speech, administrators continued to remove subreddits that broke site-wide rules as Reddit's popularity and notoriety grew. For example, in June 2013, $\mathrm{r} / \mathrm{n}^{* * * * *} \mathrm{~s}$ was banned for brigading and vote manipulation [70], and in September 2014, r/TheFappening, a subreddit dedicated to posting stolen nude celebrity photographs was banned for copyright infringement. In 2015, Reddit's administrators developed their first site-wide anti-harassment policy in which users who experienced harassment were encouraged to contact Reddit's admin; however, the announcement did not outline what, if any, sanctioning harassers would face. ${ }^{1}$ Also in 2015, Reddit introduced an additional measure to mitigate the impact of controversial subreddits: quarantining. Among other sanctions, quarantined subreddits remain on Reddit, but do not appear in non-subscription based feeds, such as r/all; are not included in searches and recommendations; are only accessible to registered users; and are labelled "Quarantined."

Despite the movement toward more active administrative sanctioning through subreddit removals, Reddit remains host to sexist, racist and xenophobic subreddits. Members of these subreddits can form what Massanari [48] describes as "toxic technocultures." Toxic technocultures propagate through networked platforms and push against values such as diversity, multiculturalism, and progressivism. Toxic technocultures are often intertwined with geek masculinity, where women are viewed as sexual objects or interlopers. Indeed, subreddits such as r/TheRedPill, r/MGTOW (men going their own way), and the recently banned $\mathrm{r}$ /braincels (involuntary celibates) promote misogyny and male supremacy. Further, a number of banned subreddits such as $\mathrm{r} / \mathrm{CreepShots} \mathrm{(sex-}$ ualized photographs of women without their knowledge), $r$ /TheFappening (hacked photographs of nude celebrities), and r/DeepFakes (fake pornographic videos of celebrities) all promoted content in which women were sexualized without their consent. While toxic technocultures can form on any platform, Massanari argues that Reddit's design affordances, such as distributed moderation, the ease of creating accounts, pseudonymity, and the hands-off governance policy of the site's administrators support the development, promotion, and proliferation of these spaces. Further, Reddit's system of upvoting provides the technical infrastructure that allows the boundaries between

${ }^{1}$ A September 30th 2019 update to the Content Policy has clarified sanctions for harassment. 
subreddits to be permeated by showcasing subreddits to users through aggregated feeds like r/all and r/popular [54]. Therefore, social norms that develop within a subreddit such as r/AskHistorians, may then be disrupted, unintentionally by new users [37] or intentionally, by brigading users [20] who may be a part of or sympathetic to the values and opinions espoused by these toxic technocultures. As this paper will demonstrate, Reddit's pattern of upvoting content that reflects the interests of the majority, in this case upvoting a question that included pictures depicting women sexualized without their consent, and expectations of hands-off governance, impact the work of moderating r/AskHistorians.

\section{METHODS}

The current work focuses on the experiences of moderators. r/AskHistorians was chosen as a case because it provides a unique opportunity to study the impact of platform technology and culture on moderation work because the subreddit's rules and norms are quite different from those of the remainder of Reddit. The following subsections describe the research site, data collection and analysis processes, and ethical considerations.

\subsection{The site: $r$ /AskHistorians}

r/AskHistorians is a large history-based question and answer subreddit. As of April 24, 2017, the day data collection began, r/AskHistorians had 593,788 subscribers and 35 users were listed as moderators. ${ }^{2}$ The subreddit was formed in August 2011 by user, $\mathrm{u} /$ Artrw with the intent of providing a space on Reddit in which curious users could ask questions and receive answers from experts in the field of history. In 2016, when subreddit subscribership rose to 500,000, r/AskHistorians moderators distributed a census; the results, based on 3893 responses, were published at the end of the year. The demographic profile captured by the census showed that subscribers were predominantly young, white, and male: the average age of census respondents was $27,81 \%$ were male, and $77 \%$ did not identify as a minority. Of the $23 \%$ who did identify as a minority, approximately half identified as being a minority based on their gender/sexuality and approximately half identified as being a minority based on their ethnicity, race, and/or nationality. Most subscribers had some level of higher education: $24 \%$ were undergraduate students, $26 \%$ had obtained an undergraduate degree, $11 \%$ were graduate students, and $13 \%$ had obtained a graduate degree; however, only $20 \%$ had or were undertaking a degree in History [36].

A key feature of $r$ /AskHistorians is its panel of experts. The panel system was established so that users could identify experts through the use of flair, a coloured line of text adjacent to the username. Those who want flair must provide evidence of their expertise by linking comments made in $\mathrm{r}$ /AskHistorians that demonstrate this expertise. Moderators review these submissions and either award flair or provide feedback on how a submission for flair could be improved.

Initially, r/AskHistorians had few subscribers and thus, few rules. However, in March 2012 subscribership grew quickly after answers to questions were shared on other, larger subreddits, thus bringing attention to the community. To ensure that responses maintained a certain standard of quality, moderators began to develop rules for asking questions and providing answers, as well as rules of conduct [24]. The rules, in brief, are:

- Be Nice: No Racism, Bigotry, or Offensive Behavior.

- Nothing Less Than 20 Years Old, and Don't Soapbox.

- Ask Clear and Specific Questions, with Time and Place in Mind.

- Write Original, In-Depth and Comprehensive Answers, Using Good Historical Practices.

$\overline{{ }^{2} \text { By September }} 2019$ membership had grown to over 1 million subscribers and the subreddit had 40 moderators. 
- Provide Primary and Secondary Sources If Asked. ${ }^{3}$ No Tertiary Sources Like Wikipedia

- Serious On-Topic Comments Only: No Jokes, Anecdotes, Clutter, or other Digressions [62].

These rules and norms allow r/AskHistorians to function as a public history site. Public history is broadly defined as "history beyond the walls of the traditional classroom" [58]. Moderator, Cassidy (u/mimicofmodes) described why $\mathrm{r} /$ AskHistorians stands out as a public history site:

... it's the most direct method of public history out there. At a living history site you have site interpreters ... between the public and the curators/researchers; a book is a one-way street, as is a museum exhibition, whether the recipient is passively taking the information that's handed out and possibly unable to get the specific information they're looking for.

By keeping discussions on topic, ensuring that responses to questions are comprehensive, that sources are provided, and that good historical practices are conducted, the mission of $\mathrm{r} /$ AskHistorians can be likened to an open, online classroom, in which anyone with interest and knowledge can contribute and learn.

\subsection{Data collection and analysis}

I collected data through observation, minimal participation, semi-structured interviews with 18 $\mathrm{r}$ /AskHistorians community members, and private messages (PMs) from an additional 4 members. Nine were moderators, three were former moderators, six were flaired users, and four had no formal role in the community (i.e., they were lurkers or occasional commenters). The current work draws from all of these interviews, but focuses on interviews and exchanges with current and former moderators. Prior to data collection I had been subscribed to r/AskHistorians for almost 5 years; however, I began formal observation of $\mathrm{r} /$ AskHistorians after I was granted permission to use the subreddit as a research site by the moderators in April, 2017 and continued until March 2018, during which time I read questions and responses, and took notes on my observations. In addition to observation of public posts, $r$ /AskHistorians moderators shared with me the full comment log, including all moderated comments, of the question transcribed in the introduction, "What is the context to these photos and was it uncommon?"

Interview participants were recruited by posting a question to r/AskHistorians asking: "Why do you read/participate in $\mathrm{r} /$ AskHistorians?" 82 people made 290 comments on the recruitment post and several more sent PMs and emails. Responses to the public post were used during analysis (users were given the option to opt out if they wanted to participate in the discussion but not the research by sending me a private message; however, none did). Other than the initial recruitment thread, my active participation in $\mathrm{r}$ /AskHistorians was minimal. While Hine [31] recommends active participation, I do not possess the requisite historical expertise to respond to questions; however, I did contribute several comments to "meta" posts (i.e., posts about the subreddit and its operation rather than history).

Those who indicated that they would be interested in participating in an interview were sent an informed consent form. Interviews were conducted via telephone or VoIP (8), video conferencing (4), face-to-face (3), or through text-based means such as synchronous instant messenger discussions (1) or through asynchronous written responses to the interview questions with emailed follow-up questions (2). Interviews lasted between 38 minutes and 3 hours and 6 minutes with an average of 1 hour and 12 minutes. See Table 1 for participant demographic information. Some demographic information is not available for the four participants who sent detailed PMs but did not participate in a formal interview.

Data analysis was conducted using a modified version of grounded theory as outlined by Strauss and Corbin [69] and Corbin and Strauss [16] in a manner similar to Thematic Analysis, as described

\footnotetext{
${ }^{3}$ While providing sources is optional, many users preemptively include sources in their replies.
} 


\begin{tabular}{|l|l|l|l|}
\hline Gender & Age & Country & Education \\
\hline & & USA:10 & Technical School: 1 \\
\cline { 3 - 4 } Female: 5 & Average: 32 & Canada: 5 & Some bachelors: 6 \\
\cline { 3 - 4 } Male: 15 & Range: $21-59$ & Australia:1 & Masters: 4 \\
\cline { 3 - 4 } & & Netherlands: 1 & Some PhD: 2 \\
\cline { 3 - 4 } & & Germany: 1 & JD: 1 \\
\hline
\end{tabular}

Table 1. Participant Demographics

by Braun and Clarke [7]. I read through the transcripts several times to identify relevant themes and concepts. As recommended by Corbin and Strauss, analysis of the data was iterative. I first read each of the interviews, and then I then engaged in several rounds of microcoding and coding to identify relevant themes and connect these themes with information gained through observation. Throughout data analysis I recorded thoughts, themes and ideas via memoing, a process that supported a dialogue between myself and the data.

\subsection{Ethical considerations}

This study was approved by the University of British Columbia's Behavioural Research Ethics Board. Nonetheless, research with human subjects often risks exposing participants to harm. To mitigate harm, those whose interview quotations and/or public comments are used in this paper were shown the quotes I intended to use, asked if they were comfortable with the use of that quote, and provided with the opportunity to amend the quote. Several participants made minor revisions to their quote(s) and/or provided information they wanted to add. Participants were also asked how they would like to be attributed. Many participants chose to use their Reddit usernames and/or first/last names as attribution. This follows procedure outlined in the Tri-council Policy Statement on the Ethical Conduct for Research Involving Humans [1], which suggests that in some circumstances participants may feel as though they could benefit from being identified in the presentation of results. Participants were also given the option to select their attribution preference for each quote; thus, a single participant may be attributed in more than one way to protect their privacy. Finally, in some cases, I was unable to reach participants. In these cases, interview participants who provided informed consent were attributed with pseudonyms, and public comments were obfuscated so that they could not be searched. Removed comments are generally quoted verbatim (including spelling, grammar, and punctuation), and without attribution to protect the privacy of those who made the comments. However, I chose to censor slurs.

Despite the above precautions, the question that runs through this paper will make some Reddit users identifiable by their usernames. The decision to use the question and thus expose identifiable usernames was not made lightly. However, it would have been difficult to tell this story without the thread: because of its co-occurrence with data collection many participants referenced the question during their interviews to illustrate the challenges they face, with some discussing it at length. Omitting reference to the thread risks minimizing key and poignant experiences described to me by my participants.

3.3.1 Position Statement. As qualitative work is a process shaped by researchers' past experiences and present state of being [17], it is important to acknowledge experiences that may shape interpretation of the data [3]. I have extensive experience using Reddit. I joined Reddit June 14, 2012, almost 5 years before I began collecting data. Shortly after joining, I subscribed to r/AskHistorians. As a Reddit user, my preference has always been for subreddits with comprehensive rules and 
active moderation. I also strongly identified with accounts that matched my pre-existing experiences as a Reddit user, in particular participants' descriptions of the effects of Reddit's culture and demographic.

\section{THE VISIBLE AND INVISIBLE WORK OF MODERATING R/ASKHISTORIANS}

Reddit's design and culture have a significant impact on the work of $r$ /AskHistorians moderators. As noted by Massanari [48] these two aspects of Reddit are inextricably combined: the design supports the culture and the culture is shaped by the design. For simplicity, the effects of each element on $\mathrm{r} /$ AskHistorians moderators is presented in a separate section. The first section provides context for the findings by providing summary of the question thread presented in the introduction; this thread will be used throughout the results to highlight various themes. The second section discusses the impact of Reddit's culture on moderation work. The third section describes the intersection of culture and design and how this impacts response quality. The fourth section focuses on how Reddit's design affects the visibility of moderation work. Finally, the fifth section describes the impact of $\mathrm{r} /$ AskHistorians on Reddit and its users. In sum, the values and demographic of the platform, combined with features which visualize moderation work that is often interpreted as censorship, make the work of public history exhausting, and may discourage participation by those with knowledge and interests outside the prevailing demographic. Despite these challenges, moderators' work makes r/AskHistorians a model community, not only as a space to promote public history, but for engaging in empathy and evidence-based knowledge production.

\subsection{The thread}

The thread opened with the question noted above, "What is the context to these photos and was it common?" It was immediately apparent that heavy oversight was given to responses as the majority were removed. Two top-level comments, both written by moderators, remained visible. The first was a "stickied" post that summarized r/AskHistorians' rules (a 'sticky' is a feature available to moderators that prevents votes from affecting the visibility of a comment, effectively "sticking" it to the top of a discussion thread). The second was a response to the question, provided by $\mathrm{u} /$ commiespaceinvader.

$\mathrm{u} /$ commiespaceinvader began his response by highlighting the difficulty of obtaining information from photographs alone, but guessed that the pictures depict members of the United States Army in Korea. While he was unable to obtain information specifically about the culture of sexual entertainment in Korea, he described similar scholarship in the context of Japan during wartime. His answer provided information about the progression of sex shows over time and the impact of cultural stereotypes. In response, the user who asked the question thanked $\mathrm{u} /$ commiespaceinvader for his answer, while another user asked if information about the location of the photographs could be gleaned by looking at the uniforms of the officers. In response to this question, $\mathrm{u} / \mathrm{commiespaceinvader}$ replied:

Frankly, I have little desire to further investigate these photos since what they depict is, when placed in the historical context of war and occupation, a public sex show intended to entertain men who compared to the women who "entertain" them are in immensely powerful positions; even up the literal power over life and death of these women. Thus what these pictures when contextualized depict is a sexual spectacle that if not outright forced, has strong implications of one party being forced to entertain another by performing sexual acts under at least indirect duress. 
This response prompted a strong reaction from users, to some $\mathrm{u} /$ commiespaceinvader responded. For example, one user questioned u/commiespaceinvader's reticence: as someone with flair denoting expertise on the Holocaust, why would sex shows be distasteful in comparison? $\mathrm{u} /$ commiespaceinvader explained that the key issue was not the material itself, but the response of the Reddit audience:

Like with the frequent questions inquiring about the blood, gore, and minutae of killing methods in the Holocaust, the distasteful part is that the interest is mere minutae, not the experience and implications behind them.

In other parts of the discussion, users questioned whether or not consent was an issue and in response, $\mathrm{u}$ /commiespaceinvader provided additional background information supporting his argument. Eventually, one of the users with which $\mathrm{u} / \mathrm{commiespaceinvader}$ was engaged in discussion conceded that their perspective had changed.

\subsection{Reddit culture and moderation work}

Responding to a post submitted in 2016 that thanked r/AskHistorians moderators for their work, moderator u/Elm11 described the impact of Reddit's culture on r/AskHistorians moderators:

the culture and popular perception of Reddit absolutely does have an impact on our activities. Reddit shapes the people who come through our door, the questions which are asked here, the issues which arise in threads on /r/all.

This comment highlights two themes that will be discussed throughout the following sections: how the upvoting system affects new users' enculturation into the community, and how Reddit's demographic (and its technology) shape the scope of the subreddit. In addition to these themes, the following sections will also discuss the impact of Reddit's culture of free speech on moderators' ability to ensure that question-askers receive high quality responses, as well Reddit users' ability to extend empathy to both historical subjects and moderators themselves.

4.2.1 Demographic effects. $\mathrm{r} /$ AskHistorians moderators highlighted a number of ways that questionasking and voting patterns of the largely white, male demographic of Reddit affected their work. First, those whose expertise falls outside the interests of the prevailing demographic described rarely having the opportunity to answer questions in their field. Further, when a question did touch on their area of expertise, it may not be from the perspective that most interested them, as was stated by Cassidy (u/mimicofmodes):

Most of my questions are about menswear (which I honestly don't care as much about as women's and children's dress), why don't we wear hats, why do we wear ties, etc. etc. While there are plenty of women who know nothing about fashion history, if there were more of them in the sub, they might at least ask about more interesting whys (when did we switch from stockings to tights, what's the history of pockets in women's dresses, did women of all classes wear corsets) - and maybe the rest of the fashion history community would be interested in asking each other questions here. ${ }^{4}$

This pattern of interest is described by moderator, $\mathrm{u}$ /sunagainstgold:

What is undeniably true, however, is the rarity of questions about women's issues (and swap in black, LGBTQ+, etc) and the patterns in which they tend to fall. Basically: rape, sex, marriage age, and rape. And rarely from women's perspective.

Similarly, when asked about the role of the demographic, moderator $\mathrm{u} /$ searocksandtrees responded:

${ }^{4}$ In a recent discussion with Cassidy she noted that this had improved since the time of the interview 
I think what it reflects to me is that there's a lot more boyish topics that come up, whether it's war and weapons and video games, and then a lot of really insensitive questions about rape.

Second, the majority of Reddit users are American, which means that questions are often asked about American history, or global history from an American perspective. For example, former moderator, $\mathrm{u} /$ Commustar described how this aspect of the demographic impacted interest in the global south:

I hoped to use my position as mod to encourage people interested in African history, South Asian history, and other under-represented areas to get involved and apply for flair. However, there was never much success attracting people to apply for flair on those regions. I think that is because questions on those regions are rarely asked, and tend to receive fewer upvotes, so there is less opportunity for knowledgeable people to comment before the posts fall off the front page and are not seen by the sub's audience. In any case, my inability to promote those sorts of discussions and find more experts was disappointing.

While he is no longer a moderator, $\mathrm{u} /$ Commustar remains an active participant on the subreddit. Although he was disappointed by his inability to recruit new members, when answering questions about Africa, u/Commustar now makes a concerted effort to decolonize citations by highlighting to $\mathrm{r}$ /AskHistorians users that Africans do engage in scholarship on their own history.

In addition to the topics covered and omitted through question asking, the way questions are asked can also impact participation. When questions are asked, they often reflect what we know, do not know, and assume about the world. Moderators and regular participants alike described learning to detect biases in question asking, often by observing more experienced community members [25]. u/Commustar provided examples of what this looks like. Questions such as, "Why was Africa less developed when Europeans started colonizing?" and "What was Nelson Mandela really like?" make certain assumptions: first about what is considered 'developed' and what is not, and second that history and the popular press present Nelson Mandela in a false light. Others ask about Africa through a Western lens, such as "what did European explorers think of the African societies they encountered?" Participants also described how biases reflected in question asking sometimes discouraged them from providing responses or continuing participation, for example, as stated by one user during a meta discussion:

African here. Most of us don't use Reddit and prefer to use a different site. Reddit doesn't have a good reputation.

I read a lot and could probably answer a few questions, but they are worded negatively and that is a turn off. It takes too much energy to dispel harmful stereotypes so I choose not to comment.

As will be discussed further below, responding to questions that reflect particular biases often results in unseen emotional labor.

In circumstances in which biased or insensitive questions are asked, moderators are tasked with making the decision to let the question stand or remove it, and experts with the decision to respond to the question or ignore it. This was the case with the, "what is the context to these photos?" thread. During our interview, moderator, Mark Evans described deliberating whether or not to remove the question:

We had a discussion about removing it because the pictures are incredibly... exploitative ... And we just felt so shitty as moderators, because here was our community, which is meant to be giving people answers about the past, but what it's doing is providing 
Redditors with porn. And that's what it ended up doing. And that's why people have ended up looking at it and it's it become a platform for these poor women to become humiliated again, like 80 years after the event. Again.

Ultimately, they made the decision to let the question stand.

4.2.2 Empathy gaps. Related to Reddit's demographic and the tendency for questions asked to reflect interests and assumptions typically held by that demographic, is what moderators referred to as an empathy gap. While moderators used this term to describe the ways in which Reddit users engage with historical subjects, interviews highlighted an additional empathy gap exhibited by users towards moderators themselves.

As noted above, questions that exhibit biases and questions that neglect particular populations are so common that moderators developed have an explanation for this phenomenon, which is outlined by moderator, $\mathrm{u} /$ sunagainstgold:

... it illustrates a distinct empathy gap, a socially-conditioned inability to default-extend intellectual personhood to people "different than us." One of the absolute most-asked questions on AH is "Did ancient soldiers have PTSD?" Sometimes we get to hear questions about knights having PTSD, too. Anyone want to take a swing at, in comparison, how many times people have asked about rape survivors and PTSD? (And when you search for it, be sure to filter out the questions that ask about the soldier-rapists developing PTSD from massacring and raping civilians).

In addition to questions that focus on men's perspectives of historical events and male subjects, questions that ask about women in history often neglect to consider their experiences. For example, rather than asking how rape as a weapon of war impacts women, questions about rape during wartime focus on quantity, such as "How many rapes were committed?"

This empathy gap is not limited to question asking; it is also reflected in responses given. However, these responses are removed by moderators and therefore largely invisible to regular users. Examples of empathy gaps exhibited toward historical subjects are peppered throughout the removed comments on the "What is the context to these photos?" thread. Examples include jokes at the women's expense, such as: "Theyre going to get sandboxes." and "A standard new England clambake, as done in French Indochina during the war." Another user made a derogatory comment about the women's breasts: "... In addition, women (and many men) were known to have an increased level of pancakes (a.ka. "flapjacks) in their diet during the war. . hence the nature of the "pancake-tits" seen in the photographs." In another example, a user refused to acknowledge that the women were likely participating due to coercion: "women are after all perfectly capable of pursuing this work out of greed and self interest." Finally, another example refers to the women as "whores." These comments neglect to treat the women as real, living people; by removing these comments and thus rendering this particular empathy gap invisible to r/AskHistorians users, moderators strive to create a safe space for women on Reddit. Moderators expressed the belief that allowing comments like these to stand (and thus potentially upvoted) would fail to exhibit compassion towards people who lived in the past.

An empathy gap is also exhibited by Reddit users toward moderators, and exists primarily in the form of online abuse. Abuse experienced by r/AskHistorians moderators ranged from mild, such as name-calling, to severe, such as prolonged harassment, doxxing, death threats, and rape threats. Mild forms of online abuse were rife in the removed comments of the "What was the context of these photos?" thread. For example, in response to a stickied comment explaining the rules of the subreddit, one user commented: "You are trying so hard to sound smart. I just imagine you as a typical 
neckbeards." Another example of abuse involved the use of a homophobic slur, "ASK HISTORIANS MODS ARE $F^{* * * *} T S$." Others expressed anger toward moderators:

What a wonderful pile of shit, I mean subreddit! So nice to come from the front page, intrigued to learn, only to be greeted by a wasteland of banned users and removed comments. Congrats dipshit mods, this subreddit is pretty much rendered worthless. Could you help me out at least once before I go? How do I add this piece of shit place to a blocked list of sorts, so that I never see it on my front page again?? Thanks!

Reddit users also responded with abusive language towards moderators providing responses. This was experienced by $\mathrm{u} /$ commiespaceinvader when he responded to the "What is the context to these photos" thread, in particular after he responded that he had no desire to delve into the minutiae of the soldiers' uniforms. While he noted that some of this abuse had come in the form of private messages, other users responded to him via the comments; for example:

Get your feelings out of history. You cant just disregard it because you dont like it. Also, that pic doesnt warrnt enough proof for it being an american insignia. Its a good thing thats just what you think.

You dont sound stable. I dont see how you observe war crimes.

and,

Christ have you ever thought about changing or removing the stick up your ass? Its sad when someone who claims to be a historian can't seem to remove his perspective and bias from 60 years later and impose it on a historical context.

Maybe these were well paid prostitutes or entertainers who were not being oppressed in their own point of view. That's likely not true but it could be. But becasue you are such a prissy uptight know it all you feel compelled to place your tight assed point of view onto it. Grow up Sheldon.

Name calling, such as the examples above, was relatively mild compared to more severe forms of abuse also experienced by $\mathrm{r}$ /AskHistorians moderators. Often, this type of abuse was targeted at specific moderators through private messaging. For example, one moderator described a particularly egregious example of abuse he had experienced:

Many of us, especially those commenting and moderating some of the more sensitive topics such as Holocaust denial or anything to do with sexism have also received unsolicited pms hurling insults at us and further. I once had a user message me 200 times in 40 minutes detailing how they would dismember and eat me after I had banned them from the sub.

One effect of Reddit's norms and culture is that it provides scaffolding for the development of bigoted communities whose members (or those who are like-minded) abuse those whose work runs contrary to their beliefs. As noted in the quote above, abuse from these groups was often topic-related; however, it was also gendered. Openly female mod, $\mathrm{u}$ /sunagainstgold expressed that she did, indeed, receive gendered abuse once harassers realize she's a woman:

Yes, I get the occasional nasty PM when it becomes apparent in a thread that I'm female-I don't hide it; I want people to know there are women hanging around.

It is likely that $\mathrm{u} /$ sunagainstgold is downplaying the quantity of gendered harassment she experiences as an openly female moderator of $\mathrm{r}$ /AskHistorians. Subsequent comments by $\mathrm{u} /$ sunagainstgold in meta threads have indicated that she has received numerous rape threads upon responding to questions on particular topics and when responding to questions in ways that call attention to 
empathy gaps towards historical subjects, particularly women. Gendered abuse led to one woman moderator I interviewed to engage in identity management and participate ostensibly as a man:

It's mostly because you get enough shit thrown at you as an $r / A s k H i s t o r i a n s$ mod without it becoming gendered. I mean I have received death threats and people threatening to murder my family not knowing that I even had a family. And I can just imagine what kind of disgusting rape comments and sexual harassment comments I would be getting if I was actually openly female. Some of the mods are openly female and I don't know how they do that.

While the majority of the moderators I interviewed described subjection to some form of abuse, their responses to abuse varied; however, a common reaction was to minimize and/or normalize it. Minimization may happen because not all abuse is equally severe. For example, some mods described finding humor in the some of the abusive messages sent:

You do occasionally get comedic ones, like the time that somebody was really mad at me and so looked at my flair and decided that I had to be a 20-something [redacted country] man living in my mother's basement and kind of dying because not only do I not meet any of those criteria, but my mother was actually living in my basement at the time! (Anna)

It also may be that it felt minimal in comparison to what was expected: "It's not as bad as I thought it might be. There's less of it that's intentional rather than simply young people who haven't been exposed to other ideas yet" (James Brooks). While minimization did occur, normalizing abuse and other negative effects of managing disruptive behaviour was more common, and is illustrated in this statement by Mark Evans:

Some of it you just get really sick of discovering how many racists we deal with on a day to day basis. It just becomes normalized. It's perfectly normal for me to see people denying the Holocaust on a daily basis, because why wouldn't you, right? Like, stuff that really shouldn't be just normal kind of is.

Other mods described tuning out abuse and other disruptive behaviour or described it as white noise. While they rarely discussed it explicitly in terms of normalization, it is possible that finding humour in, and minimizing the effects of disruptive behaviour are normalization tactics that help moderators cope with negative aspects of their work.

\subsection{Culture and design: Ensuring quality in the context of Reddit-style free speech}

Ensuring that question-askers receive high quality responses to their questions is the primary objective of r/AskHistorians and the core rationale for the development and enforcement of the subreddit's rules. The rules are published in several places on the subreddit: in brief in the sidebar of the subreddit's page and in depth in its wiki. In addition to listing the rules, the wiki defines each rule, describes forms of sanctioning for violating the rule, and links to round table discussions explaining why each rule was developed. Further, when questions become popular, moderators summarize and provide a brief rationale for the rules in a comment stickied to the top of the popular discussion thread.

The combination of sparse site-wide rules and guidelines and cultural ideas of free speech means that some Reddit users, including those who subscribe to r/AskHistorians, are unsupportive of rules that are perceived to limit speech, and view sanctioning as a form of censorship. This attitude is displayed in several removed comments in the "What is the context to these photos" thread. For example, the conflation of moderation and censorship is apparent in this comment: "11,000 upvotes. All the comments are deleted because of censorship. This post is a ghost town. Reddit is dead. R.I.P". Further, those who view moderation as censorship may perceive the censors as dictatorial, a theme 
that can be seen in another removed comment from the thread: "Mods on this sub read too mucb about Hitler, huh?"

Of the hundreds of removed comments in the thread, only a minority conflate sanctioning and censorship. However, the majority did not understand why so many comments were removed, asking a variation of, "where are all the comments?" For example, this comment, which asked: "wtf happened to all the comments here?" While others did not reference censorship, they did express dissatisfaction with the moderation style, for example: "Everything is banned on this subreddit? What is this cancer mod work?" As strict rules and enforcement are not the norm across Reddit, Reddit users who discover $\mathrm{r}$ /AskHistorians for the first time when a question hits the front page or who have subscribed to r/AskHistorians without knowing, understanding, or supporting its rules, often expect to see participation as they would on other subreddits and may continue to participate on the subreddit as they would on others. The expectation that $r$ /AskHistorians moderation will follow Reddit-wide norms and be decentralized is often expressed in meta threads (i.e., posts about the subreddit itself), and was also seen in a comment made in response to the study recruitment post: "I'd just like to see all the answers and let community votes do the decision making, personally."

$\mathrm{r} /$ AskHistorians moderators described a variety of reasons why they opposed using the karma system as an indication of quality. First, the majority of those who upvote responses do not have the requisite expertise to evaluate quality; second, voting reflects user bias; and third, earlier comments tend to receive more upvotes, regardless of quality. As ensuring question-askers receive high quality responses is the core goal of the subreddit, moderators see their role in removing low quality responses as a responsibility supporting this goal:

A major part of that effort has been to delete comments that are low effort, or speculative, or promote pseudohistory or bigotry. The AH mod team sees the deletion of such bad comments as "curating the sub", akin to pulling out weeds so flowers can grow (u/Commustar).

One of the reasons why moderators view removing comments as the key to achieving this goal is because they have observed that Reddit users have the tendency to upvote responses that reflect a poor or incomplete understanding of history. Two interview participants described incidents in which comments that they believed should have been removed but were not were then highly upvoted, such as flaired user, MrDowntown (Dennis McClendon):

But something that I've encountered a couple of times in the last year to my frustration is that I won't see a question for three or four hours and then somebody once had a college class that read a chapter about this topic will have given what I would consider a Canswer. Something that is only tangential to the central question that's been asked, but by the time I get to the question they have been upvoted 30, 40 times, and my, what I think is a better answer, eh, 8 or 10 people see it.

The problem of high quality responses hidden by low quality ones was also described by readers as frustrating. For example, when describing why he likes r/AskHistorians, Matt (a lurker) highlighted the importance of removing low quality responses:

I don't want to have to search through a bunch of people making Alexander the Great puns. I like going in and seeing one really good post from a flaired commentator ... and then a whole bunch of crap deleted underneath - that's beautiful! This is a wonderful part of the Internet!

As is reflected in his first sentence, good information can be difficult to find amid jokes and other comments that neglect to fully or reliably respond to the question.

Further, moderators also expressed concern that allowing users to determine what they think is the best response by upvoting could promote and propagate harmful misinformation: 
The frequently brought up argument that the ideas of Holocaust deniers will be easily defeated in the "free market place of ideas" is to me as someone who deals with the subject an incredibly misguided one since: A.) lying is always easier than debunking lies. People who deny the Holocaust will simply say „,crematoria don't produce smoke! it is all a lie; and for those debunking them, it is necessary to actually make an argument based around how crematoria actually work, which is not something most of us have ever expected to deal with. And B.) it assumes that all people are rational and will follow the better argument (hello again, white, male, patriarchal notion of knowledge), which as current politics illustrate is decidedly not the case. People will believe what fits their world view. (u/commiespaceinvader)

Another issue (also reflected in u/commiespaceinvader's statement) is that not only may nonhistorians not be able to fully assess the quality of responses, but that voting often reflects users' biases. This was also observed by Mills [54], who, in his study of political advocacy on Reddit, found that highly upvoted comments often reflect users' consensus on a given topic. In a system where voting determines what content is seen and what is hidden, and where voting often reflects bias, the biases held by the prevailing demographic are those that will be promoted.

\subsection{Reddit's design and visible and invisible moderation work}

By design, moderation work on Reddit is both visible and invisible. This section will focus on some of the design elements that impact moderation work by making it visible or invisible.

4.4.1 Visible work: comment removal. As part of an effort to maintain transparency, Reddit's design makes comment removal visible by replacing removed comments that have received a response with the epitaph, "[removed]." In addition to labelling removed comments, the total number of comments made is displayed at the top of each post, rather than the total that remain, and is visible to users before clicking on the post. Because moderators actively enforce the rules, posts typically contain very few remaining responses; thus, there are often far fewer visible comments than the number displayed by the system. The incongruity between the number displayed and the actual number of visible comments causes confusion for users, particularly those who are unfamiliar with $\mathrm{r}$ /AskHistorians and expect to see a certain number of comments prior to clicking on a post. Users' confusion is apparent in the removed comments of the "What is the context to these photos?" thread. By the time the post had run its course, Reddit users left 723 comments. However, of the 723 total comments, only 39 remained. As the question garnered upvotes, new users who were unfamiliar with $\mathrm{r}$ /AskHistorians rules and norms saw the thread and commented en masse. In an attempt to explain the rules to new users, moderator, $\mathrm{u}$ /sunagainstgold stickied a comment providing an overview of the rules, the rationale for creating them, and a warning that they would be enforced. Nonetheless, users continued to post rule-breaking comments. Moderator, $\mathrm{u} / \mathrm{E} \operatorname{lm} 11$ responded to the stickied comment to highlight the content of the removed comments:

Well, by my count, which I'm sure will be out by a few, of the 594 comments in this thread (595 including this post), 534 of them are just asking or complaining about the removed comments.

That's right, a whopping eighty nine percent of you (of course, some will have posted before the pinned to level, but most are much more recent) either didn't take the time to read this top level or just plain old didn't care and wanted to add to the pile. This is the worst thread I've ever seen for "WHERE ARE TEH COMMENTS" spam, so y'all have that going for you. Of the remaining 60 comments, the vast majority are one liners, with a smattering of 
slightly longer (but still insufficient) speculative responses, with a few mod posts thrown in.

Basically, this thread looks like an enormous graveyard because of all of the drongos having a whine about how much of a graveyard it is. If you'd stop belly-aching you all have some patience and wait for a quality answer to arrive, as they do in the overwhelming majority of popular threads, the thread wouldn't be as ugly and empty as it is.

\section{Thanks for your time.}

The frustration expressed by $\mathrm{u} / \mathrm{Elm} 11$ is palpable. The complete comment log shows that comments asking a variation of "where are all the comments?" range from innocent queries by new users who do not understand $\mathrm{r}$ /AskHistorians rules, e.g., "Are there usually so many removed comments here? I'm just popping in from $/ \mathrm{r} /$ all, I don't frequent this subreddit." to expressions of disappointment from users who understand the rules, but do not support them, e.g.,

boooo. i'd rather have some speculation or anecdotes than [deleted]x500. but i'm just a lowly non-historian from r/all. sorry for adding to your workload by giving you another comment to delete. it's lonely here.

to insulting, e.g., "Damm is there anyone whose comment you haven't removed, douche." Showing how many comments are removed and highlighting their removal maintains transparency, which can allow users to make informed decisions about how they interact with the site [22] and impact trust [39]. However, it also creates additional work for moderators. The visibility of removing comments creates a feedback loop, where people who see that comments have been removed leave even more comments that break the rules, thus necessitating the removal of more rule-breaking comments. Further, because the work visibly sanctions users, it also exposes moderators to harassment and abuse from those who have been sanctioned, as discussed earlier.

While comment removal is visible, the work associated with removal and the impact of exposure to removed comments is invisible. Several moderators I interviewed described feeling shocked after becoming a moderator, describing the transition from reader (or flair) to moderator as a paradigm shift, such as $\mathrm{u}$ /searocksandtrees: "I suddenly saw that this civilized, grown up, friendly corner of the internet I found wasn't actually that at all! It was just as crap as everywhere else." To reduce this work, moderators have employed technical workarounds, such as programming Automoderator to filter certain comments so that they can more quickly review comments that are likely to be rule-breaking and, more recently, working with a community member to launch a browser plugin that displays the number of remaining comments rather than the total number of comments.

While new and occasional users expressed dissatisfaction with $\mathrm{r}$ /AskHistorians moderation style in the removed comments of the "What is the context to these photos?" thread, it is likely that most regular $\mathrm{r} /$ AskHistorians readers support the rules and their enforcement. In both the census distributed by moderators and in my interviews with non-moderators, $r$ /AskHistorians moderation style was widely supported. In the census $91.6 \%$ respondents believed the moderators' efforts were just about right. The general consensus that $\mathrm{r} /$ AskHistorians' rules support its mission is likely a contributing factor to its growth [38].

4.4.2 Invisible work: responding to questions. Just as Reddit's design makes some work visible, it also renders other work invisible. In many cases, $r$ /AskHistorians moderators are also subject experts; thus, much of their work includes responding to questions. Because the upvoting system works quickly, at times launching posts to the front page within minutes, users often see questions when they are popular, rather than answers when they are given. While it can take very little time for a popular question to be upvoted to the front page of Reddit, it can take a long time to write a 
comprehensive response to a question. During interviews, some moderators reported responding to questions outside of their area of expertise, thus requiring time to search for and read relevant information in addition to writing a response. For example, one moderator described biking to a nearby library to access a paywalled journal article he needed to respond to a question while another described collaborating with other moderators on Slack to search for and access potentially relevant articles. The disconnect between the time it takes for a question to reach the front page and the time it takes to write a response is demonstrated in a removed comment from the "what is the context to these photos?" thread: "6 hours $9 k$ upvotes. pitty it doesnt look like anyone can provide an answer to OP. i guess that's an accomplishment? have something unique enough to stump everyone!"

The "What is the context to these photos?" question was particularly difficult to answer. During our interview, $\mathrm{u} /$ commiespaceinvader expressed concern that the speed at which the question was being upvoted and the time of day it was posted (nighttime in the United States) meant that it was unlikely to get answered by someone with expertise in the area. While $\mathrm{u} /$ commiespaceinvader had written answers on sexual violence during wartime in the past, he did not consider this topic within his area of expertise. After responding to the question, he described spending the rest of the day reading about sexual violence and sexual entertainment during wartime to be better equipped to quickly respond to similar questions he anticipated receiving in the future. In addition to the challenge of quickly writing a response to a question outside his area of expertise, $\mathrm{u}$ /commiespaceinvader also described the emotional labor involved with responding to the question, particularly in response to the pushback he received: "It can be difficult to maintain a semi-professional tone etc. when one is confronted with upright hostility because of the content of my answers or because of moderation." To manage this emotional labor, $\mathrm{u} /$ commiespaceinvader reached out to other moderators via backchannels, where he had a safe space to discuss his frustration.

4.4.3 Invisible work: maintaining community health. Community building and evaluation is another example of invisible work undertaken by moderators. Building and maintaining community health takes a variety of forms, such as developing ways to increase participation and quantitative assessments of the community. For example, popular threads often contain complaints that questions never get answered. To determine the extent of this claim, moderator u/Georgy_K_Zhukov analyzed the top 50 posts each month over the course of two years to find the average response rate (which was 96\%). Moderators also distribute and analyze semi-regular censuses to learn more about subreddit users. Upon becoming a panelist, flaired users are granted access to a private subreddit, which is moderated by the same moderator team as $\mathrm{r}$ /AskHistorians, although interviewees did note that moderating this subreddit required little time and effort. Several moderators also described acting as information brokers. Because they have a good sense of where expertise lies in the community, moderators noted that they would alert those with expertise in a given area when a relevant question was asked. They also respond to modmail queries and private messages, which could range from explaining why a comment or question was removed, to providing feedback on how to improve that comment or question, to recommending books and other sources, to providing professional advice. Finally, evaluating the quality of answers was also described as a form of invisible work. Deciding whether an answer to a question should remain or be removed sometimes requires a degree of expertise in the topic area. Interviewees noted that if the quality of an answer was in question, they would discuss whether it should be removed or remain with other moderators on the team. 


\subsection{The impact of $\mathbf{r}$ /AskHistorians}

The work conducted by moderators, both visible and invisible, has an impact. First, their labor is performed in the service of public scholarship and second, the unique rules and their enforcement creates what can be perceived as a model community on Reddit.

4.5.1 Public scholarship. One of the key motivations for participation described by moderators was the mission of the subreddit: public history [25]. r/AskHistorians performs this role by providing a space in which interested users can ask questions, read responses, and engage in discussions about history. The result is that the community provides an important two-way space for learning, where an interested audience can connect with experts who want to share. While it was not directly stated as the rationale for allowing the "What is the context to these photos?" thread to remain on the subreddit, it is likely that the opportunity for learning that the thread presented was the reason why it was not removed. Learning through participation in the subreddit was also described as a key motivation by users with a variety of roles, including readers, flaired users, and moderators [25]. The rules, and moderators enforcement of those rules, were described by lurkers as an integral part of learning as the rules helped establish trust in information shared on $\mathrm{r} /$ AskHistorians. For example, lurker, Jon, compared r/AskHistorians to r/history, which has laxer rules: "one of the reasons I like going to [r/AskHistorians] is because it's a lot more trustworthy than something that is on, like r/history, where anyone can do whatever they want." The rules and their enforcement were also described as an integral part of sustained participation: "If the mods decided to loosen up the rules I probably wouldn't read as much. Their quality control is very important” (Reagan).

Because it shares a platform with communities that promote and spread conspiracy theories and disinformation, such as Holocaust denial, r/AskHistorians' rules and mission as a public history site also place the subreddit and its expert users in a position to directly address issues like Holocaust denial. As noted above, Reddit is host to subreddits that promote white supremacy, bigotry, and conspiracies. As Reddit users are not limited to participation in particular subreddits, users from these subreddits, or users exposed to the ideologies promoted on these subreddits, may also participate in $\mathrm{r}$ /AskHistorians. Because Holocaust deniers need a public forum to sow doubt and because of their unwillingness to engage with historic facts, $r$ /AskHistorians bans Holocaust denial [8]. When questions are asked that touch on common Holocaust denial talking points, moderators assess whether or not the question is asked in good faith or is an attempt to promote Holocaust denial through a tactic known as "Just Asking Questions," or JAQing off; the former are allowed to stand while the latter are removed. To respond to good faith questions, moderators developed a pre-written response that addresses common Holocaust-denial talking points to ensure that question-askers are provided with information.

Finally, in addition to providing a space in which information is exchanged, $r$ /AskHistorians also provides a space in which empathy, particularly towards historical subjects, is taught and learned. For example, in my recruitment post, one r/AskHistorians user commented:

Answers here have helped me inform my political opinion, my thoughts regarding issues such as LGBT rights and feminism (it was actually an answer here that made me fully consider patriarchy theory!), colonialism and and its very subtle effects on today's society, and last but perhaps most importantly, have had an influence on my overall thought process and problem solving.

Another example of learning empathy can also be seen in the "What is the context to these photos?" thread: 


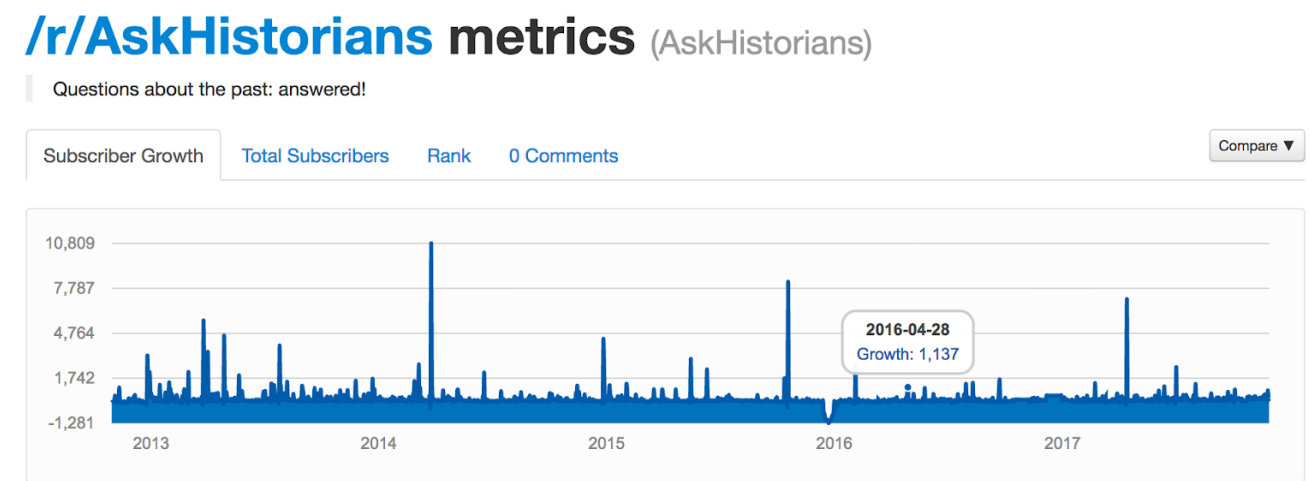

Fig. 1. AskHistorians subscriber growth

I can say with absolute certainty that you changed my perspective on the topic at hand. What I saw as a relatively harmless consensual situation was revealed to be something very different which I would have never considered.

Through engagement with $\mathrm{u} / \mathrm{commiespaceinvader,} \mathrm{this} \mathrm{user} \mathrm{was} \mathrm{encouraged} \mathrm{to} \mathrm{question} \mathrm{their}$ assumptions and focus on the experiences of the women who were the subject of the photographs rather than the men watching them.

4.5.2 A model community. Although $\mathrm{r} /$ AskHistorians location on Reddit is, at times, problematic for the subreddit, there are also advantages. While highly upvoted posts expose the community to disruptive users en masse, upvoting also exposes these new users to r/AskHistorians. Figure 1 shows the impact highly upvoted posts have on subscribership ${ }^{5}$, where the spikes correlate with posts that hit the front page of Reddit; the third spike depicts subscribership growth after the "What is the context to these photos?" question.

Mills [54] describes how ranking posts by upvotes creates a positive feedback loop because other users mimic what they see upvoted. In response to this phenomenon, moderators created a "Great Question" flair to encourage patterns in question asking and upvoting. To further mitigate the impact of voting patterns, regular features, such as Monday Methods, meta posts, and more recently, Floating Features have been created by moderators to highlight pertinent issues and allow people to respond to questions that may not arise organically through question-asking.

$\mathrm{r}$ /AskHistorians' success as a public history site may not only have a positive effect on individual users, but as described by $\mathrm{u} /$ restricteddata, on the rest of Reddit and online communities more broadly:

AH is sort of a "killer app" for "what the Internet could be if people are willing to put the effort into it" and I think that's very positive. The fact that the rest of Reddit can be so awful in so many different ways only underscores the contrast - if Reddit can be made to be non-awful, what else is possible in the world?

Indeed, Chandrasekharan et al [13] used $\mathrm{r}$ /AskHistorians as an example of a well moderated site in an attempt to identify abusive behaviour using machine learning techniques. r/AskHistorians rules are derived from norms established within academic history and modified to include a broader

\footnotetext{
${ }^{5}$ Subscribership data included in the image does not include mobile users.
} 
audience. Thus, the exact style or approach may not work for all other subreddits or communities. However, in carving out a regulated space in which readers can access trustworthy information about the past, and engage with it through question-asking, follow-up, and debate, r/AskHistorians is an apt model for promoting civil discourse online and at scale.

\section{DISCUSSION}

\subsection{How Reddit's design affects volunteer moderation work}

Platform policy and design dictates the transparency of moderation. On platforms that rely heavily on paid moderation, such as Facebook, this work is almost entirely invisible to users, save for those whose content is removed. The result is a largely seamless interaction with information where content is viewed as a natural phenomenon and values motivating the removal of content are hidden [64]. When this invisibility is made opaque, such as the revelation that content displayed on social media platforms does not reflect everything that is shared, this is often met with distress, for example, as can be seen in the public's reaction to the Facebook emotional contagion study, where the public perceived Facebook and the researchers as manipulating the content to which they were exposed [28] or the public's reaction to human moderators tagging news content [9].

Reddit relies on several levels of moderation: administrators, who have the ability to ban communities and users from the site; volunteer moderators, who have the ability to remove content and ban users from their subreddits; and Reddit users, who engage in distributed moderation through the practice of upvoting and downvoting. As multiple actors engage in moderation work, Reddit provides an infrastructure that enables the development of localized norms and values within subreddits. Nonetheless, technological features, such as distributed moderation, and a culture of free speech contribute to overarching values and norms that pose challenges to volunteer moderators of subreddits, like $r$ /AskHistorians, that operate under different values. When visible moderation work, such as removing comments, defies site-wide norms, those doing that work must manage disruptive behaviour and are exposed to pushback and abuse. The more upvotes a post receives, the more disruption and abuse moderators must manage. Hiding certain types of moderation work can prevent exposure to abuse. For example, the ability to ban or sanction rule-breaking users from a generic moderator account can minimize the effects of violent backlash by reducing rape threats, doxxing, and sustained harassment campaigns. However, visible work, such as responding to questions in ways that humanizes subjects, has shown to be an effective method of teaching and encouraging users to extend empathy toward historical subjects. Could visible work be leveraged so that users can extend empathy towards moderators?

Recent work focusing on the viewpoints of those whose content has been moderated showcase the importance of transparency in moderation [32, 33, 57]. For example, in her study of Facebook users, Myers [57] found that the gap between the actual cause of removal and why users think their content has been removed, resulted in the development of 'folk theories', where users developed their own explanations for the effects of actions within technical systems [18]. Others found fundamental normative disagreements between what users and moderators perceived as transgressions [4, 34, 35] Recent empirical studies exploring the impact of providing explanations for removal have shown that users who were provided with explanations were more likely to accept the removal, and that providing explanations reduced the number of future transgressions [32, 33].

Communicating rules and norms by posting rules and providing explanations are examples of work r/AskHistorians moderators alternatively make visible and invisible. Among the visible work is sharing the rules, which are posted in several places with links to round table discussions that provide a detailed rationale for each of the rules. In popular threads, moderators take an additional step to communicate subreddit rules via summarizing them in a comment stickied to the top of the 
thread. At times, they may also use this space to highlight the content of removed messages, to ensure users that removed comments do not contain sufficient responses to the question. Some moderators chose to list or count these messages while others have provided screenshots showing examples of removed comments. In the example of the "What is the context to these photos?" the removed comments show that users continued to post comments asking why the thread had been so heavily moderated, even after the rules had been stickied. While the stickied comment may have reduced the total number of questions and comments than the question would have received without the stickied comment [52], it did not solve the problem entirely and resulted in additional emotional labor. Further, while making this work visible may have had a positive impact on users participating in good faith, it may have worked to further expose moderators to surveillance from bad actors, who used the opportunity to make abusive comments under the assumption that they would be removed. Future work should explore qualitative responses to moderator communication to explore ways to encourage the extension of empathy toward moderators and suggest best practices for moderator/user communication.

The trade off between visibility and communicating rules and norms highlights the importance of invisible backchannels. In discussing his experience responding to the "What is the context to these photos?" question, u/commiespaceinvader acknowledged the important role relationships with and support from fellow moderators played in helping him express his frustration while maintaining a professional tone in his responses. In their work on HeartMob, a platform built by and for people who experience online harassment, Blackwell et al [5] describe the important role a backchannel can play for those subjected to online abuse to safely discuss their experiences and receive social support. For some HeartMob users, the act of labelling what they had experienced as abuse made it visible, provided validation, and helped resist the normalization of abuse. r/AskHistorians moderators have several backchanels including a private subreddit and a Slack channel. Future work on the use of backchannels could provide more insight into how making work visible to a controlled audience of trusted friends and colleagues; i.e., selective visisbility $[10,21]$ can mitigate the more negative aspects of volunteer moderation.

\subsection{How Reddit's culture affects volunteer moderation work}

Free speech is embedded in Reddit's culture. While there has been a movement by its administration from unabated free speech to limiting speech via banning or quarantining subreddits, many Reddit users continue to value and expect speech free from sanctioning. A partial explanation may be found in Reddit's rules and guidelines and their somewhat lackadaisical enforcement. While these can be found in two places, its Content Policy and Reddiquette respectively, specific sanctioning for violating guidelines is specified for three activities: posting personal information, off-Reddit requests for votes, and spamming. While these broad guidelines have the advantage of allowing considerable freedom to establish how subreddits are run, it also means that subreddits themselves are not required to have rules beyond the guidelines established by the site. Indeed, in their study characterizing Reddit's rules, Fiesler et al. [23] found that $48 \%$ of their sampled subreddits did not have any posted rules, suggesting a laissez faire approach to community management. Gillespie [26] notes that a site's rules are demonstrative of its performed ethos. Reddit's lassez faire approach to sanctioning demonstrates an ethos of lawlessness; when lawlessness is framed as 'free speech,' moderation is seen as censorship, a phenomenon demonstrated in the removed comments from the "What is the context to these photos?" thread.

In discussing the right to look, Mirzoeff [55] states: "The opposite of the right to look is not censorship ... but visuality, that authority to tell us to move on and that exclusive claim to be able to look." Visuality, according to Mirzoeff, is an act of power in which those who wield it control what is seen, and through it, manifest their authority. At first glance, it seems at though

, Vol. 1, No. 1, Article . Publication date: February 2020. 
this is the role of $r$ /AskHistorians moderators, who control what is seen on the subreddit through question and comment removal. The perception of comment removal as a revocation of users' right to look at comments (and that removed content is thus "censored") is supported by both Reddit's culture of free speech and its design, where distributed moderation obfuscates downvoted comments by collapsing them rather than removing them entirely. However, Mirzoeff describes visuality as an act undertaken by slavers, generals, police, and politicians, metaphors that do not aptly describe $r$ /AskHistorians moderators and their work. If $r$ /AskHistorians moderators control what is seen, but their work does not align with the above listed professions, what might be a more appropriate metaphor? The mission of public history makes r/AskHistorians' work more akin to that undertaken by instructors. Instructors control what information is allowed in their classroom, not to maintain power, but to ensure the best possible educational experience for learners. Indeed, Mirzoeff describes education as a means of emancipation-as part of seeing. In addition to removing content that has little educational value, $r$ /AskHistorians moderators also leverage visuality to make the subaltern visible and close empathy gaps by decolonialzing history, countering misinformation rooted in bigotry, and resisting normative narratives around gender and sexuality; for example, when $\mathrm{u}$ /commiespaceinvader re-centered the focus of the "What is the context to these photos?" question from the uniformed men to the women.

The educational service provided by $\mathrm{r}$ /AskHistorians has a positive impact on Reddit and its users. Nonetheless, $r$ /AskHistorians moderators face challenges that result from a divergence between norms that are enabled through the platform's design. To close empathy gaps between moderators and users, particularly in learning-centric spaces, platforms should leverage norms (e.g., academia) and metaphors (e.g., instructors) that more aptly describe the work moderators do and will help users better understand moderator actions.

\subsection{Inverting design and culture to foster trust}

Massanari's [48] work demonstrates the role Reddit's design, in particular the karma system, plays in promoting and perpetuating norms and values held by the majority of users while silencing the voices of marginalized populations. r/AskHistorians showcases how moderators can use rules and norms to partially subvert this model. Reddit's design still affects r/AskHistorians: upvotes reflect interest in particular types of questions (as can be seen in the interest shown by a majority male audience to a question containing nude photographs of women), tend to favour responses made earlier, drive traffic from users unfamiliar with the subreddit's rules, and the nebulous boundaries between subreddits allow bad actors to participate. However, through the creation of rules and alternative norms, $\mathrm{r}$ /AskHistorians moderators push against dominant norms of free speech to ensure that question-askers receive comprehensive responses to questions containing information that users trust is accurate. This is in large part due to rules surrounding sourcing, where respondents who do not preemptively supply sources must do so if asked; comprehensiveness, where pithy comments are removed to allow time and visibility for more comprehensive responses to be written; and preparedness, where responders must be able to answer follow up questions.

In writing about how media literacy has failed, danah boyd [6] describes how her privilege as an academic has allowed her to see how knowledge is produced and understand the strengths and weaknesses of scientific investigation. Through its rules and norms, $r$ /AskHistorians extends existing models of public scholarship that focus on information dissemination, open access, and knowledge translation to an instructional model of information literacy that builds readers' skills, such as learning historical methods. The community also teaches readers how to identify and account for bias in source material [25], and the discussions in which users read and engage provide rich opportunities for learning in the wild [29,41]. 
In addition to the discussions that remain on the subreddit, many of which provide viewpoints that encourage the development of empathy or the inversion of common assumptions, r/AskHistorians' moderation work also serves to counteract the impact of subreddits created to promote disinformation based on misogyny, bigotry, racism, and antisemitism. This work is two-fold: first, by removing questions and comments that promote hateful rhetoric, and second, by providing explanations and feedback to users who are likely asking poorly worded questions in good faith. Much of this type of work is intentionally invisible to the broader audience. $r$ /AskHistorians moderators argue that a free marketplace of ideas will not result in a consensus based on truth and fact [8]; rather, people will draw conclusions based on prior assumptions and biases [2]. As Levitt et al [45] found, Reddit users perceive upvoted content to be more trustworthy. In r/AskHistorians, the audience often does not have the expertise to judge a response and experts within the community have found that leaving responses open to the free marketplace of ideas risks validating information based on prior misconceptions about particular aspects of history. In cases where questions and comments promote ideologies based on hate, such as Holocaust denial, moderators have written detailed scripts that preemptively address common talking points. In this way, question-askers are provided with information, but hateful ideologies are not propagated. This highlights a delicate balance that builds on work by Jhaver and colleagues [32,33]; while providing public explanations for removal may reduce moderation work and improve the relationship between the users and moderators, moderators who wish to employ such explanations would also need to take into account any mission or goals of their subreddit and identify when public explanations can work to support goals and when they risk subverting them. In the case of $r$ /AskHistorians, public explanations for certain infractions can bring undue attention to ideologies based on disinformation and grounded in hate.

\subsection{Limitations and future work}

This work is limited in that it focuses on the perspective of moderators and participants who generally have a favorable view of the subreddit. While the viewpoints of other users are included through the discussion of removed comments, these participants were not interviewed and thus their viewpoints and motivations are not equally represented in this work. As a case study, the impact of Reddit's design and culture may affect moderators of other communities differently; further, as $r$ /AskHistorians is driven by a mission of public history, approaches to moderation that work for $\mathrm{r}$ /AskHistorians moderators may not be translatable to communities with other goals or that have particular professional guidelines, such as attorneys and physicians. Future research on other subreddits can provide insight into these remaining questions.

\section{CONCLUSION}

Volunteer moderators play key roles in online spaces as they create and enforce rules and model normative behavior. Using an ethnographic approach, this study explored moderation work in r/AskHistorians, an online community with a mission of promoting public history. As a public history site, $r$ /AskHistorians operates under rules and norms that differ from those of the wider platform. Thus it is an apt case to study the impact of platform design and culture on moderation work. Results showed that visible moderation is often interpreted as censorship. This, coupled with the default masculine whiteness of Reddit, create challenges for moderators, such as encouraging empathy towards historical subjects and encouraging participation by marginalized populations. Nonetheless, $r$ /AskHistorians moderators have carved a space on Reddit where, through its public scholarship work, the community serves as a model for combating misinformation by building trust in academic processes. 


\section{ACKNOWLEDGMENTS}

There are several groups whose contributions to this work were invaluable. First, I would like to thank Caroline Haythornthwaite, Katie Shilton, and Mark Evans for reading drafts of this work and providing thoughtful feedback. Second, I would like to thank the r/AskHistorians moderator team for opening their community to me, taking the time to discuss their experiences with me, and for continuously supporting my work. Finally, I would like to thank Johannes Breit, whose intellectual generosity is unbounded.

\section{REFERENCES}

[1] 2018. Tri-Council Policy Statement: Ethical Conduct for Research Involving Humans - TCPS 2 (2018). Retrieved October 10, 2019 from http://www.pre.ethics.gc.ca/eng/policy-politique_tcps2-eptc2_2018.html

[2] Hunt Allcott and Matthew Gentzkow. 2017. Social media and fake news in the 2016 election. Fournal of economic perspectives 31, 2 (2017), 211-36.

[3] Roni Berger. 2015. Now I see it, now I don't: Researcher's position and reflexivity in qualitative research. Qualitative research 15, 2 (2015), 219-234.

[4] Lindsay Blackwell, Tianying Chen, Sarita Schoenebeck, and Cliff Lampe. 2018. When online harassment is perceived as justified. In Twelfth International AAAI Conference on Web and Social Media.

[5] Lindsay Blackwell, Jill Dimond, Sarita Schoenebeck, and Cliff Lampe. 2017. Classification and its consequences for online harassment: Design insights from heartmob. Proceedings of the ACM on Human-Computer Interaction 1, CSCW (2017), 24.

[6] danah boyd. 2017. Did media literacy backfire?

[7] Virginia Braun and Victoria Clarke. 2006. Using thematic analysis in psychology. Qualitative research in psychology 3 , 2 (2006), 77-101.

[8] Johannes Breit. 2018. How One of the Internet's Biggest History Forums Deals With Holocaust Deniers.

[9] Matt Carlson. 2018. Facebook in the news: Social media, journalism, and public responsibility following the 2016 trending topics controversy. Digital journalism 6, 1 (2018), 4-20.

[10] Matthew Carrasco and Andruid Kerne. 2018. Queer Visibility: Supporting LGBT+ Selective Visibility on Social Media. In Proceedings of the 2018 CHI Conference on Human Factors in Computing Systems. ACM, 250.

[11] Alissa Centivany. 2016. Values, ethics and participatory policymaking in online communities. In Proceedings of the 79th ASIS\&T Annual Meeting: Creating Knowledge, Enhancing Lives through Information \& Technology. American Society for Information Science, 58.

[12] Eshwar Chandrasekharan, Mattia Samory, Shagun Jhaver, Hunter Charvat, Amy Bruckman, Cliff Lampe, Jacob Eisenstein, and Eric Gilbert. 2018. The Internet's Hidden Rules: An Empirical Study of Reddit Norm Violations at Micro, Meso, and Macro Scales. Proceedings of the ACM on Human-Computer Interaction 2, CSCW (2018), 32.

[13] Eshwar Chandrasekharan, Mattia Samory, Anirudh Srinivasan, and Eric Gilbert. 2017. The bag of communities: identifying abusive behavior online with preexisting internet data. In Proceedings of the 2017 CHI Conference on Human Factors in Computing Systems. ACM, 3175-3187.

[14] Adrian Chen. 2011. Misguided Internet Vigilantes Attack College Student's Cancer Fundraiser.

[15] Adrian Chen. 2012. Reddit CEO speaks out on violentacrez in leaked memo:'We stand for free speech'.

[16] Juliet Corbin and Anselm Strauss. 2008. Basics of qualitative research: techniques and procedures for developing grounded theory. Thousand Oaks, California: Sage.

[17] Norman K Denzin and Yvonna S Lincoln. 2008. Introduction: The discipline and practice of qualitative research (3 ed.). Sage, $1-32$.

[18] Michael A DeVito, Jeremy Birnholtz, Jeffery T Hancock, Megan French, and Sunny Liu. 2018. How people form folk theories of social media feeds and what it means for how we study self-presentation. In Proceedings of the 2018 CHI Conference on Human Factors in Computing Systems. ACM, 120.

[19] Julian Dibbell. 1994. A rape in cyberspace or how an evil clown, a Haitian trickster spirit, two wizards, and a cast of dozens turned a database into a society. Ann. Surv. Am. L. (1994), 471.

[20] Bryan Dosono and Bryan Semaan. 2019. Moderation Practices as Emotional Labor in Sustaining Online Communities: The Case of AAPI Identity Work on Reddit. In Proceedings of the 2019 CHI Conference on Human Factors in Computing Systems. ACM, 142.

[21] Brianna Dym, Jed R Brubaker, Casey Fiesler, and Bryan Semaan. 2019. " Coming Out Okay" Community Narratives for LGBTQ Identity Recovery Work. Proceedings of the ACM on Human-Computer Interaction 3, CSCW (2019), 1-28.

[22] Motahhare Eslami, Kristen Vaccaro, Min Kyung Lee, Amit Elazari Bar On, Eric Gilbert, and Karrie Karahalios. 2019. User Attitudes towards Algorithmic Opacity and Transparency in Online Reviewing Platforms. In Proceedings of the 
2019 CHI Conference on Human Factors in Computing Systems. ACM, 494.

[23] Casey Fiesler, Joshua McCann, Kyle Frye, Jed R Brubaker, et al. 2018. Reddit rules! characterizing an ecosystem of governance. In Twelfth International AAAI Conference on Web and Social Media.

[24] David Fouser. 2017. A short history of AskHistorians (from someone who was there). (2017). National Council on Public History Annual Meeting.

[25] Sarah Ann Gilbert. 2018. Motivations for participating in online initiatives: exploring motivations across initiative types. Ph.D. Dissertation. University of British Columbia.

[26] Tarleton Gillespie. 2018. Custodians of the Internet: Platforms, content moderation, and the hidden decisions that shape social media. Yale University Press.

[27] James Grimmelmann. 2015. The virtues of moderation. Yale fL \& Tech. 17 (2015), 42.

[28] Blake Hallinan, Jed R Brubaker, and Casey Fiesler. 2019. Unexpected expectations: Public reaction to the Facebook emotional contagion study. New Media \& Society (2019), 1461444819876944.

[29] Caroline Haythornthwaite, Priya Kumar, Anatoliy Gruzd, Sarah Gilbert, Marc Esteve del Valle, and Drew Paulin. 2018. Learning in the wild: coding for learning and practice on Reddit. Learning, Media and Technology 43, 3 (2018), 219-235.

[30] Susan Herring, Kirk Job-Sluder, Rebecca Scheckler, and Sasha Barab. 2002. Searching for safety online: Managing" trolling" in a feminist forum. The information society 18, 5 (2002), 371-384.

[31] Christine Hine. 2008. Virtual ethnography: Modes, varieties, affordances. The SAGE handbook of online research methods (2008), 257-270.

[32] Shagun Jhaver, Darren ScottC Appling, Eric Gilbert, and Amy Bruckman. 2019. "Did You Suspect the Post Would be Removed?": Understanding User Reactions to Content Removals on Reddit. Proc. ACM Hum.-Comput. Interact 1, 1 (2019).

[33] Shagun Jhaver, Amy Bruckman, and Eric Gilbert. 2019. Does Transparency in Moderation Really Matter?: User Behavior After Content Removal Explanations on Reddit. Proceedings of the ACM on Human-Computer Interaction 2 (2019).

[34] Shagun Jhaver, Larry Chan, and Amy Bruckman. 2017. The view from the other side: The border between controversial speech and harassment on Kotaku in Action. arXiv preprint arXiv:1712.05851 (2017).

[35] Shagun Jhaver, Sucheta Ghoshal, Amy Bruckman, and Eric Gilbert. 2018. Online harassment and content moderation: The case of blocklists. ACM Transactions on Computer-Human Interaction (TOCHI) 25, 2 (2018), 12.

[36] jschooltiger. 2016. Census 500K Results. Retrieved October 10, 2019 from https://www.reddit.com/r/AskHistorians/ comments/5fx1xc/census_500k_results/

[37] Charles Kiene, Andrés Monroy-Hernández, and Benjamin Mako Hill. 2016. Surviving an eternal september: How an online community managed a surge of newcomers. In Proceedings of the 2016 CHI Conference on Human Factors in Computing Systems. ACM, 1152-1156.

[38] Sara Kiesler, Robert Kraut, Paul Resnick, and Aniket Kittur. 2012. Regulating behavior in online communities. Building successful online communities: Evidence-based social design (2012), 125-178.

[39] René F Kizilcec. 2016. How Much Information?: Effects of Transparency on Trust in an Algorithmic Interface (pp. 2390-2395).

[40] Peter Kollock and Marc Smith. 1996. Managing the virtual commons. Computer-mediated communication: Linguistic, social, and cross-cultural perspectives (1996), 109-128.

[41] Priya Kumar, Anatoliy Gruzd, Caroline Haythornthwaite, Sarah Gilbert, Marc Esteve del Valle, and Drew Paulin. 2018. Learning in the wild: Coding Reddit for learning and practice. In Proceedings of the 51st Hawaii International Conference on System Sciences.

[42] Cliff Lampe and Paul Resnick. 2004. Slash (dot) and burn: distributed moderation in a large online conversation space. In Proceedings of the SIGCHI conference on Human factors in computing systems. ACM, 543-550.

[43] Cliff Lampe, Paul Zube, Jusil Lee, Chul Hyun Park, and Erik Johnston. 2014. Crowdsourcing civility: A natural experiment examining the effects of distributed moderation in online forums. Government Information Quarterly 31, 2 (2014), 317-326.

[44] Jean Lave, Etienne Wenger, et al. 1991. Situated learning: Legitimate peripheral participation. Cambridge university press.

[45] Alex Leavitt and John J Robinson. 2017. The role of information visibility in network gatekeeping: Information aggregation on Reddit during crisis events. In Proceedings of the 2017 ACM conference on computer supported cooperative work and social computing. ACM, 1246-1261.

[46] Claudia Claudia Wai Yu Lo. 2018. When all you have is a banhammer: the social and communicative work of Volunteer moderators. Master's thesis. Massachusetts Institute of Technology.

[47] Alice Marwick. 2012. The public domain: Surveillance in everyday life. Surveillance \& Society 9, 4 (2012), 378-393.

[48] Adrienne Massanari. 2017. \# Gamergate and The Fappening: How Reddit's algorithm, governance, and culture support toxic technocultures. New Media \& Society 19, 3 (2017), 329-346. 
[49] J Nathan Matias. 2016. The civic labor of online moderators. In Internet Politics and Policy conference, Oxford, United Kingdom. 1-10.

[50] J Nathan Matias. 2016. Going dark: Social factors in collective action against platform operators in the Reddit blackout. In Proceedings of the 2016 CHI conference on human factors in computing systems. ACM, 1138-1151.

[51] J Nathan Matias. 2019. The civic labor of volunteer moderators online. Social Media+ Society 5, 2 (2019), 2056305119836778.

[52] J Nathan Matias. 2019. Preventing harassment and increasing group participation through social norms in 2,190 online science discussions. Proceedings of the National Academy of Sciences 116, 20 (2019), 9785-9789.

[53] Aiden R McGillicuddy, Jean-Gregoire Bernard, and Jocelyn Ann Cranefield. 2016. Controlling Bad Behavior in Online Communities: An Examination of Moderation Work. (2016).

[54] Richard A Mills. 2018. Pop-up political advocacy communities on Reddit. com: SandersForPresident and The Donald. AI \& SOCIETY 33, 1 (2018), 39-54.

[55] Nicholas Mirzoeff. 2011. The right to look. Critical Inquiry 37, 3 (2011), 473-496.

[56] Kevin Morris. 2011. Reddit shuts down r/jailbait.

[57] Sarah Myers West. 2018. Censored, suspended, shadowbanned: User interpretations of content moderation on social media platforms. New Media \& Society 20, 11 (2018), 4366-4383.

[58] National Council on Public History. 2018. What is public history?

[59] Elinor Ostrom. 2000. Collective action and the evolution of social norms. Fournal of economic perspectives 14, 3 (2000), 137-158.

[60] Hector Postigo. 2003. Emerging sources of labor on the Internet: The case of America online volunteers. International review of social History 48, S11 (2003), 205-223.

[61] Hector Postigo. 2009. America Online volunteers: Lessons from an early co-production community. International fournal of Cultural Studies 12, 5 (2009), 451-469.

[62] r/AskHistorians. 2019. r/AskHistorians. Retrieved October 10, 2019 from https://www.reddit.com/r/AskHistorians/

[63] Howard Rheingold. 1993. The virtual community: Finding commection in a computerized world. Addison-Wesley Longman Publishing Co., Inc.

[64] Sarah T Roberts. 2019. Behind the Screen: Content Moderation in the Shadows of Social Media. Yale University Press.

[65] Joseph Seering, Tony Wang, Jina Yoon, and Geoff Kaufman. 2019. Moderator engagement and community development in the age of algorithms. New Media \& Society (2019), 1461444818821316.

[66] Aaron Shaw and Benjamin M Hill. 2014. Laboratories of oligarchy? How the iron law extends to peer production. Journal of Communication 64, 2 (2014), 215-238.

[67] Tim Squirrell. 2019. Platform dialectics: The relationships between volunteer moderators and end users on reddit. New Media \& Society (2019), 1461444819834317.

[68] Susan Leigh Star and Anselm Strauss. 1999. Layers of silence, arenas of voice: The ecology of visible and invisible work. Computer supported cooperative work (CSCW) 8, 1-2 (1999), 9-30.

[69] Anselm Strauss and Juliet Corbin. 1998. Basics of Qualitative Research (2nd. ed.). Sage, Thousand Oaks, CA.

[70] Bridget Todd. 2013. Does Anything Go? The Rise and Fall of a Racist Corner of Reddit. 